\title{
A METHOD OF FOREFOOT RECONSTRUCTION
}

\author{
Alan W. Fowler, Bridgend, Wales
}

The operation to be described is designed for the treatment of the severest claw toe deformity when it is impossible to restore the normal anatomy of the metatarso-phalangeal joints, and when there is severe pain under the prominent metatarsal heads. The operation is not intended to replace any of the established conservative operations for claw toes such as tenotomy, tendon transfer, capsulotomy, arthrodesis of the interphalangeal joints and proximal phalangectomy.

Etiology-Twelve of the twenty patients with irreversible clawing described here suffered from the destructive effects of rheumatoid arthritis. The remaining patients had claw toes associated with hallux valgus or pes cavus, in whom prolonged fixed deformity had caused disorganisation of the metatarso-phalangeal joints.

Morbid anatomy-The toes are drawn on to the dorsum of the foot and the metatarsal heads are prominent in the sole. This prominence is partly due to the piston-like action of the proximal phalanges which depress the metatarsal heads (Fig. 1). However, it is a mistake to assume that the metatarsal heads will be restored to their normal position when toe pressure is eliminated, whether by amputation of the toes (Fig. 2) or by the provision of adequate toe room in the shoes.

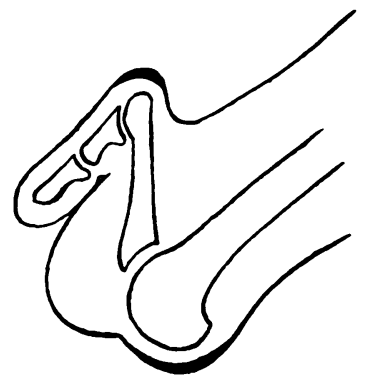

FIG. 1

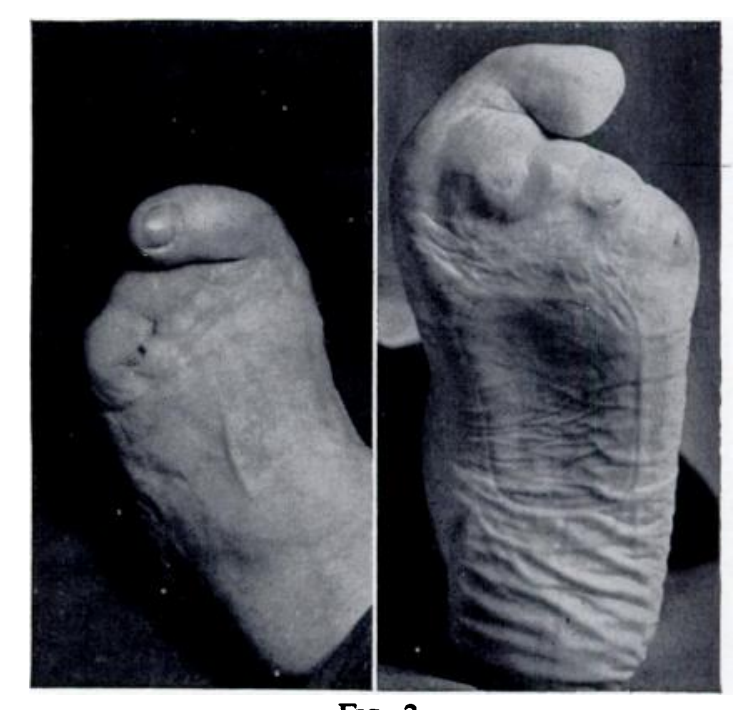

Fig. 2

Figure 1-Diagram showing the mechanism by which the proximal phalanges depress the heads of the metatarsals into the sole of the foot. The metatarsal weight-bearing pad is drawn forwards and lies in front of the metatarsal heads. Figure 2Amputation of the toes has not relieved pressure on the metatarsal heads in this foot.

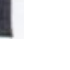


for a short distance along the shafts of the first and fifth metatarsals (Fig. 4). The skin, veins, extensor tendons and fat are divided and reflected in one layer to expose the metatarsophalangeal joints. The proximal halves of the proximal phalanges are removed, care being taken not to injure the flexor tendons.

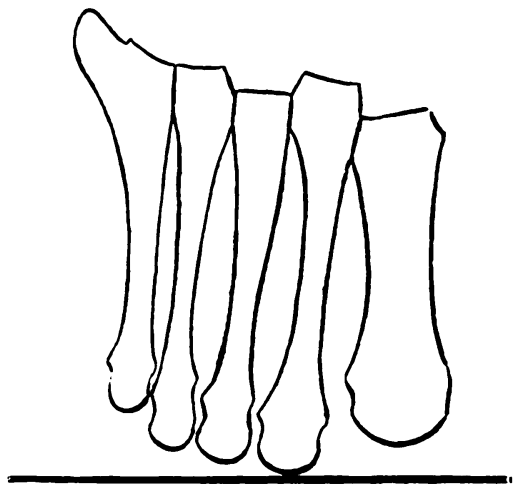

FIG. 3

The extra length of the second, third and fourth metatarsals renders them more prominent when the foot is in a shoe with a raised heel and when the foot is in the " take off" position.

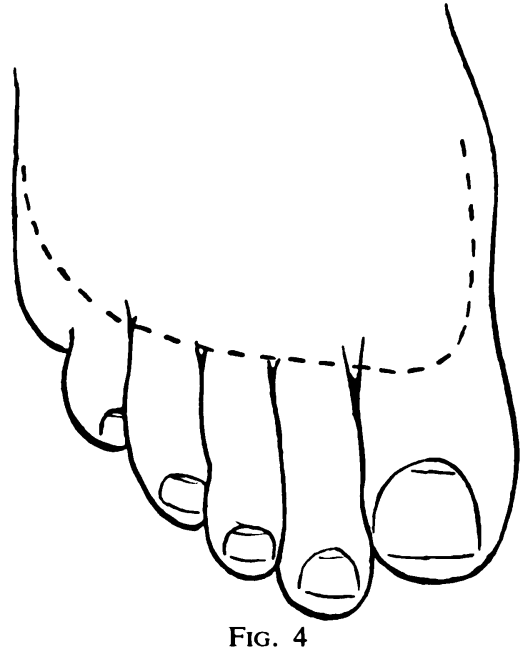

The incision which divides skin, veins and extensor tendons.

The metatarsal heads are now exposed and their alignment can be determined (Fig. 5). For reasons stated above it is important to reduce the length of the longer metatarsals so as to straighten the convex anterior line of the metatarsal heads (Fig. 6). The under surfaces of the metatarsal heads are trimmed so as to form an even weight-bearing area. At the least, the condylar projections are removed, but it is usually necessary to remove more than this

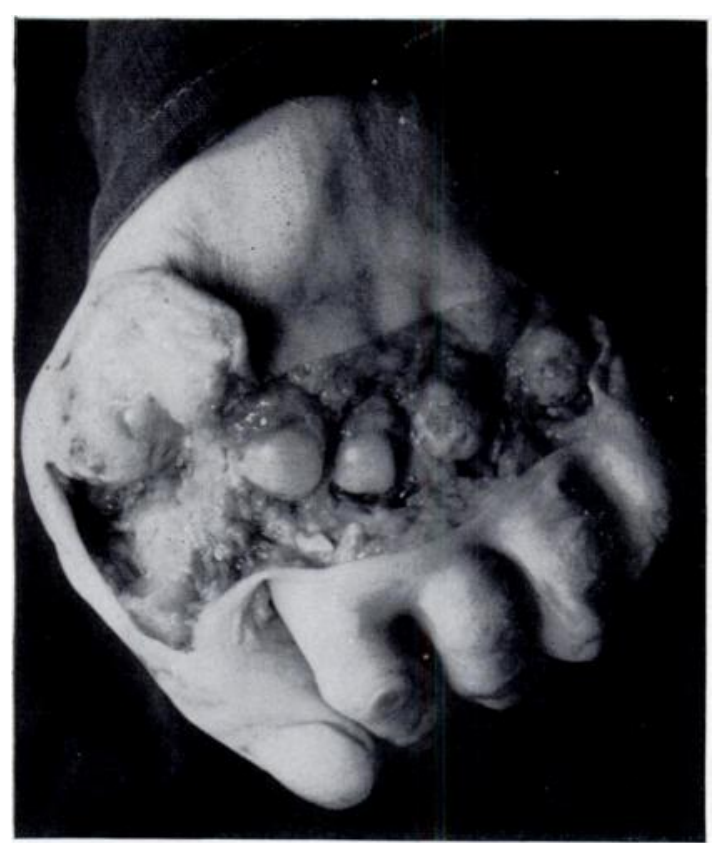

FIG. 5

The exposure of the metatarsal heads after excision of the proximal halves of the proximal phalanges. from some heads in order to bring all the metatarsals to the same level (Fig. 7). In severe cases it may be necessary to excise the whole of the metatarsal heads, and further shortening of the metatarsals may be required in pes cavus. In reshaping these bones it is important to bear in mind that the weight-bearing surface will not lie parallel with the shaft of the metatarsal but at a variable angle depending on the height of the longitudinal arch and of the heel of the shoe (Fig. 8).

Usually the sesamoids will retract when the base of the proximal phalanx is removed, thus reducing the relative prominence of the first metatarsal, unless they are adherent, when they must be excised.

The skin alone is sutured, and the toes return to their deformed position with the metatarsal weight-bearing pad still displaced forwards (Figs. 8 and 9). To correct this deformity an ellipse of skin is removed from the plantar surface of the foot behind the 
metatarsal heads. Enough skin is removed so that when the wound is sutured the metatarsal pad is replaced beneath the remodelled metatarsal heads (Figs. 10 and 11). It must be emphasised that only the thickness of the skin is removed, as in taking a Wolfe graft.

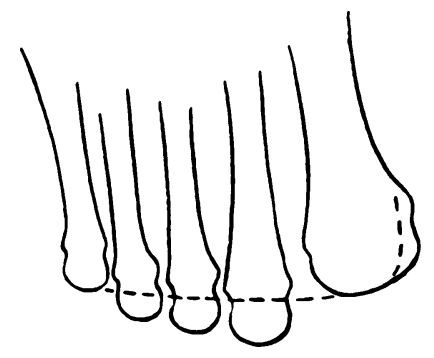

FIG. 6
Fig. 6

Trimming the metatarsal heads. The longer metatarsals are shortened so that the convex anterior line of the metatarsals is straightened.

\section{FIG. 7}

Sufficient bone is removed from the plantar surfaces to produce a uniform weight-bearing surface.

A pressure dressing is applied; no plaster fixation is used because swelling after operation is considerable. After two to three weeks the sutures are removed and the toes are then carefully aligned in a plaster toe cap (Fig. 12). This is worn for four weeks and the patient is encouraged to walk. After removal of plaster an insole incorporating a metatarsal bar is worn in a low-heeled shoe.

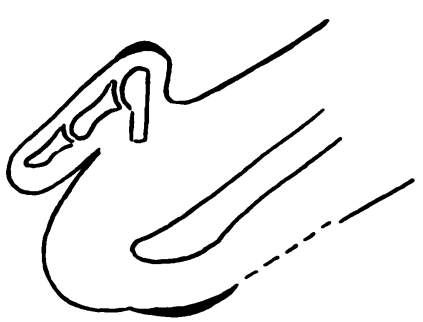

FIG. 8

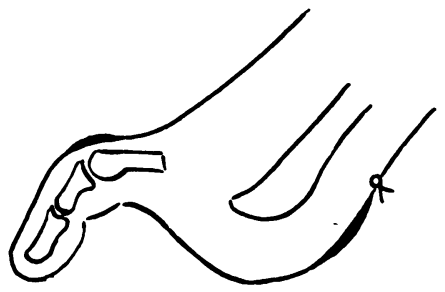

FIG. 10

Figures 8 to 11 -Diagrams showing how the metatarsal weight-bearing pad is replaced beneath the remodelled metatarsal heads by removing an ellipse of skin from behind the metatarsal heads.

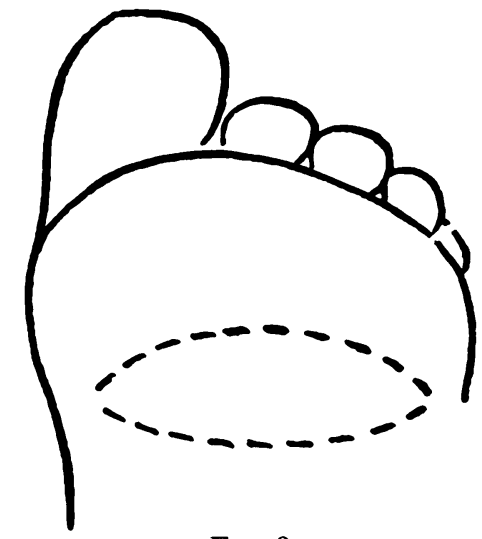

Fig. 9

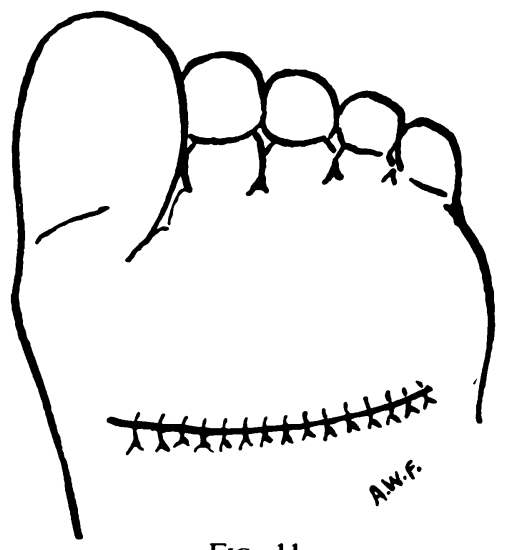

Fig. 11

This operation can be modified to suit any particular patient; thus, if the toes are not severely deformed the removal of bone can be limited to the metatarsal heads and the excision of sole skin can be omitted. On the other hand if the deformity at the proximal interphalangeal 
joints is severe it may be necessary to excise more than half of the proximal phalanges. If the alignment of the toes is satisfactory plaster fixation is not always necessary but it does help to control swelling during early walking.

\section{RESULTS}

This paper reports the results of forefoot reconstruction on the first twenty patients, fifteen of whom had the operation on both feet. The follow-up period varies from one to four and a half years, the average being two years three months. All patients expressed gratitude for gaining great relief from pain and for a new lease of activity (Figs. 13 to 16).

When re-examined, seven patients were found to have one or more small plantar callosities but these were either painless or caused little discomfort. The first patient operated on was an exception; she required further operation on both feet in order to trim away more bone. Exposure of the metatarsal heads through the old incision was accomplished without difficulty.

The recurrent callosities did not appear until more than six months after operation, and all occurred in patients who had not been supplied after operation with metatarsal supports. This is the reason why a metatarsal support is prescribed even though most do not feel the

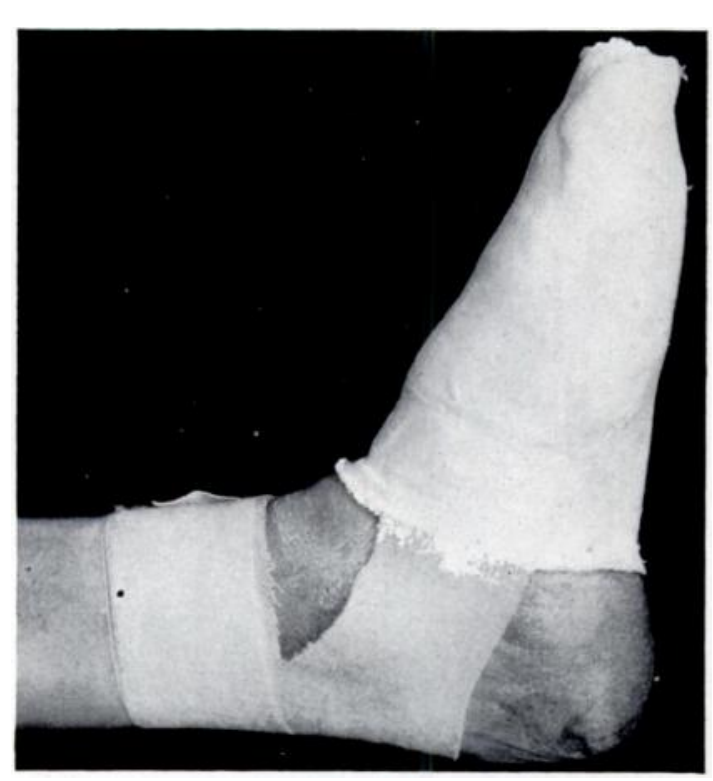

Fig. 12

A plaster toe cap may be worn during the first few weeks of walking. need for it. The remodelled heads cannot be as efficient as the normal and it is therefore reasonable to continue with efforts to relieve pressure from them.

In the early operations the metatarsal heads were trimmed too sparingly; with increasing experience more bone has been removed. One patient, however, illustrated a possible disadvantage of removing the whole of all the metatarsal heads. This was a woman who had had some difficulty in her balance. After the operation she felt more unsteady and tended to fall forwards, possibly because of the shortening of the foot.

In one patient the return of circulation after release of the tourniquet was sluggish and the edge of the proximal flap died. This patient had atrophic tissues and a tourniquet should not have been used. The skin on the dorsum of the foot has an inferior blood supply; so the proximal flap should be dissected as little as possible.

In one patient the sutures were removed on the tenth day and the wounds gaped. The sutures should be retained for two weeks on the dorsum of the foot, and up to three weeks on the sole.

\section{DISCUSSION}

This is a preliminary communication. Final appraisal of this operation can be possible only after ten or twenty years of "wear" on the feet. But, if the operaton is reserved for severe deformities, it is unlikely to do harm and the relief will probably be permanent. Forefoot reconstruction should be a last resort-a salvage operation when other measures have failed to give relief. The operation does not always eliminate the need for surgical shoes, especially in those patients with feet deformed by rheumatoid arthritis.

It is usually recommended that if a patient with irreversible clawing of the toes cannot be made comfortable in surgical shoes then the last resort is amputation of all the toes and remodelling of the metatarsal heads (Nissen 1957). Provided the metatarsal heads are trimmed, 
amputation gives good results, but forefoot reconstruction has two advantages. Firstly, the metatarsal weight-bearing pad is replaced, whereas after amputation of the toes it is drawn farther away on to the dorsum of the foot. Secondly, the cosmetic result is more acceptable;
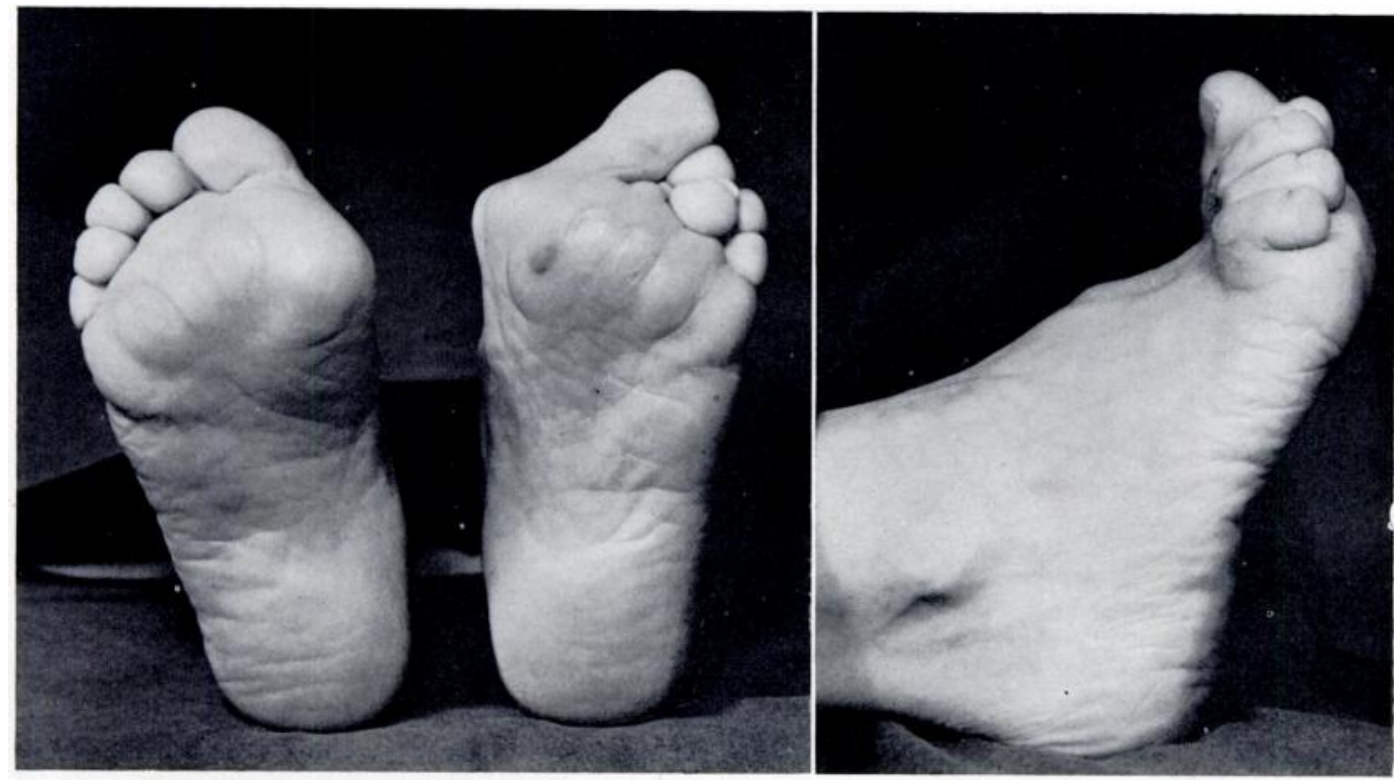

FIG. 13
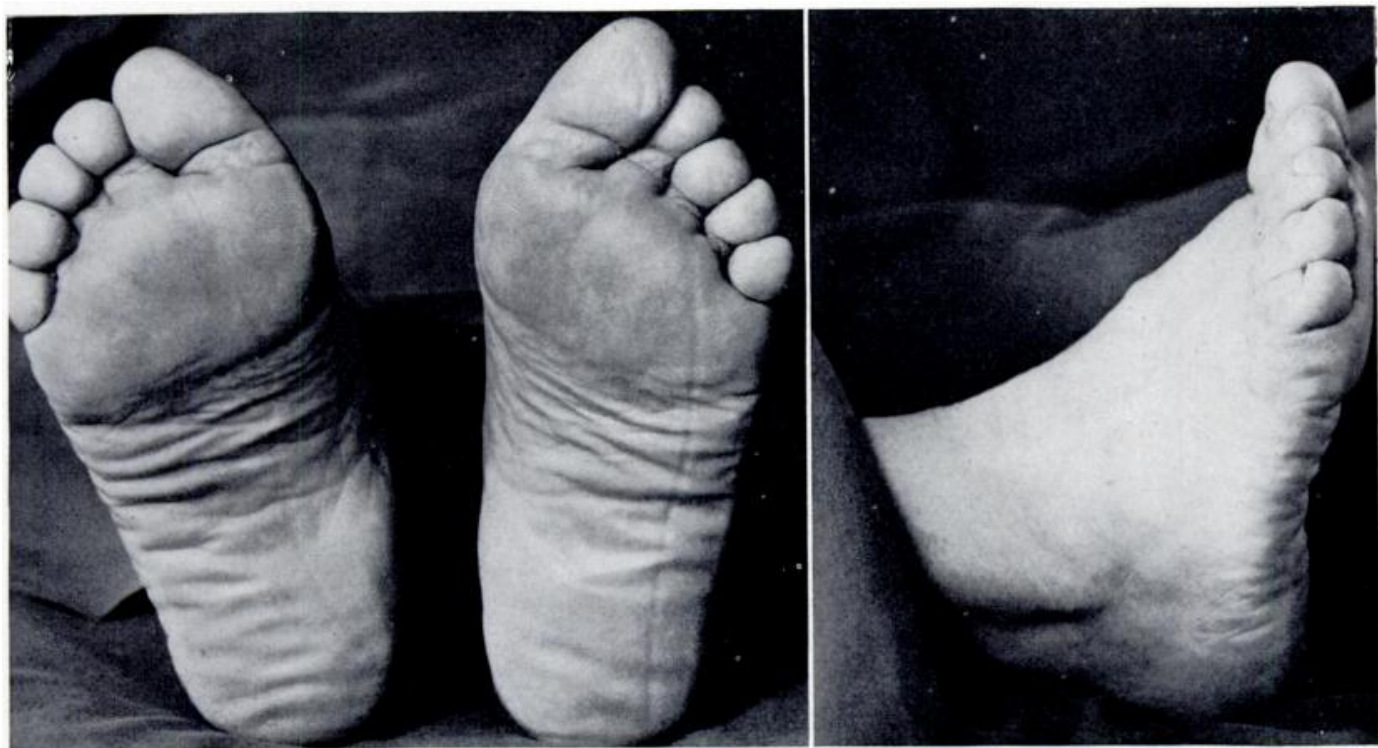

FIG. 14

Figure 13-The feet of a woman aged fifty-one with rheumatoid arthritis. Figure 14-The appearance sixteen months after operation.

patients will readily agree to have their toes straightened but they are reluctant to have them removed.

It is freely admitted that the toes have little active function after forefoot reconstruction, but they are not entirely useless because, being partly flail, they readily conform to, and fill up, the empty space at the front of the shoe. In this respect the toes resemble the hallux after a vol. 41 B, No. 3, AUguSt 1959 
successful Keller's operation with the hallux assuming the valgus position necessitated by most types of footwear. In spite of this the bunion does not recur because the metatarsal head, in addition to being trimmed, is disconnected from the proximal phalanx which can
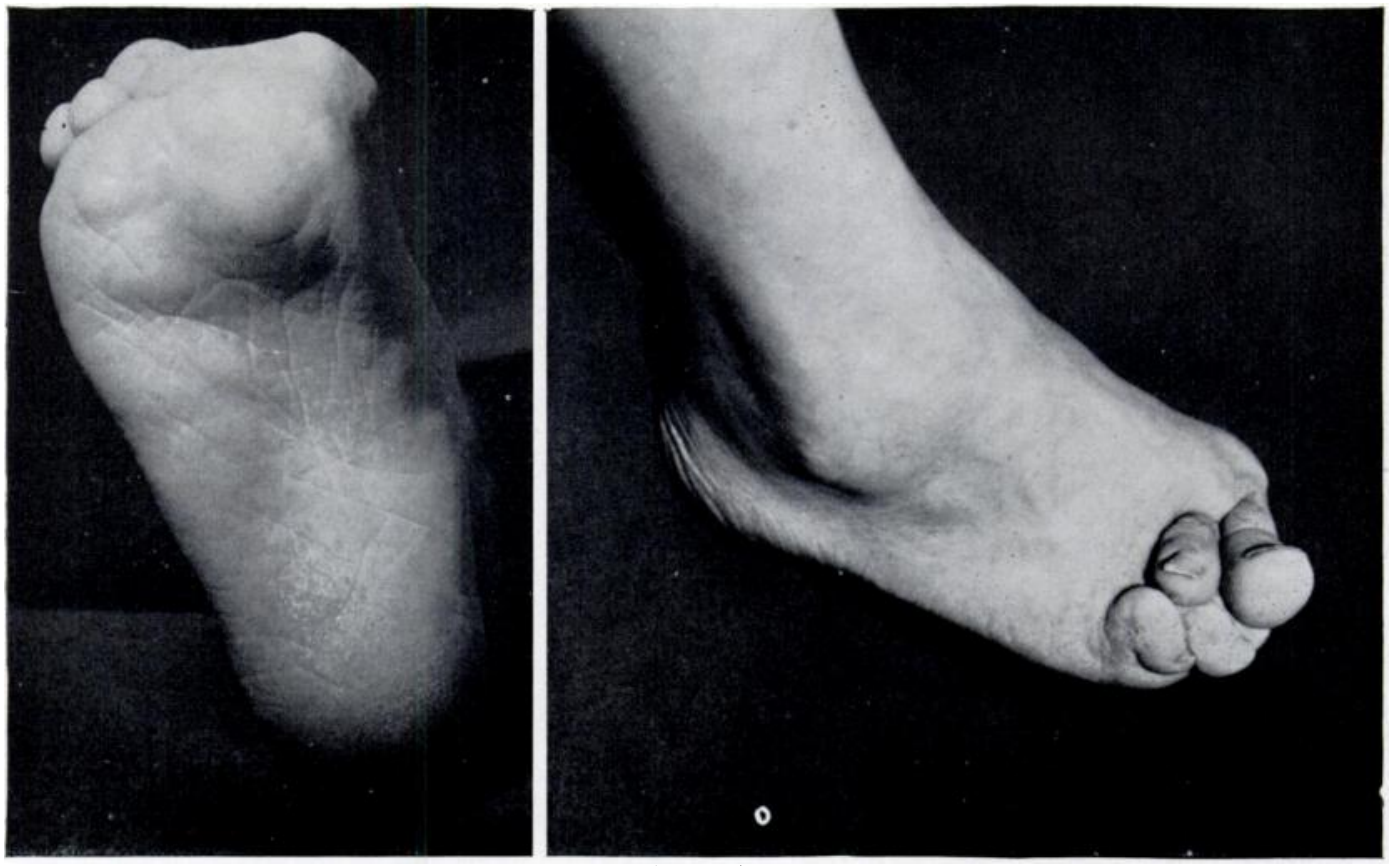

Fig. 15
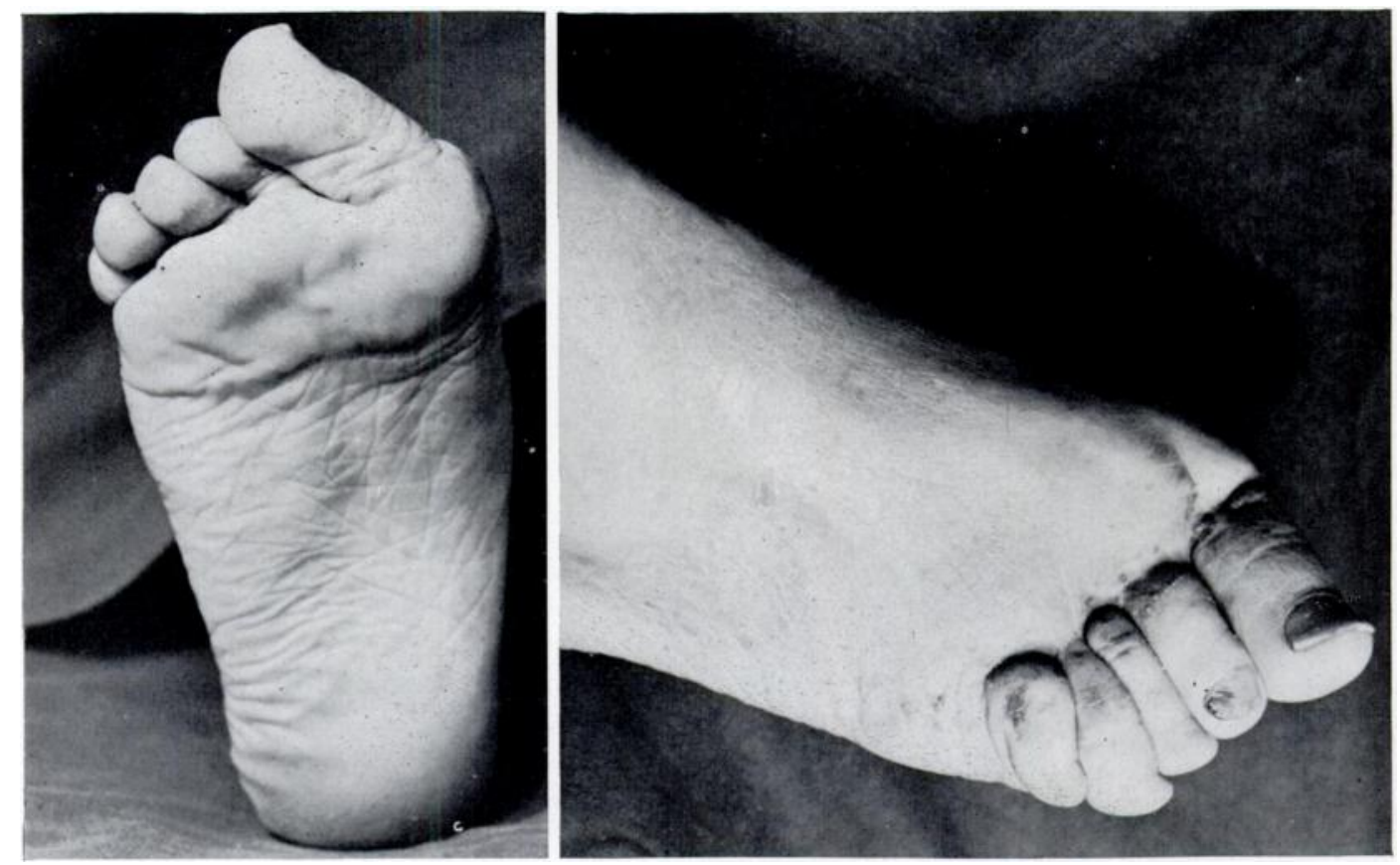

FIG. 16

Figure 15-The right foot of a woman aged fifty-four with rheumatoid arthritis. Figure 16-The appearance twenty months after operation.

THE JOURNAL OF BONE AND JOINT SURGERY 
no longer impart a medial thrust to the metatarsal head. Forefoot reconstruction involves a similar procedure on all the toes, providing relief from pressure by the removal of prominent bone and the disconnection of the proximal phalanges from the metatarsal heads.

The dorsal transverse approach has several advantages. It divides no important structures, provides easy access to the metatarsal heads, is repaired by skin sutures only, and leaves an inconspicuous scar. Two other approaches to the metatarsal heads have been described. Hoffmann (1912) advocated resection of the metatarsal heads through a transverse plantar incision behind the webs of the toes. With this approach there is a possibility of damage to the neurovascular bundles and flexor tendons. Another disadvantage of Hoffmann's operation is that failure to remove any of the proximal phalanx calls for wider excision of the metatarsal in order to correct the dorsal displacement of the toes.

Larmon (1951) described an approach using three separate incisions. A Keller's operation is done on the hallux, and the other metatarsal heads are reached through two dorsal longitudinal incisions in the webs between the second and third and the fourth and fifth toes. This is a difficult approach because of the limited space and the depth of the metatarsal heads. Another disadvantage is that because the metatarsal heads are not all exposed in the same wound their relative prominence cannot be determined and therefore it is difficult to decide how much bone to remove.

Finally, mention should be made of two operations designed to relieve prominent metatarsal heads without removing bone from them. McKeever (1952) described a metatarsal shortening operation for the treatment of plantar callosities. The necks of one or more metatarsals are divided and the heads replaced after shortening the shafts. Broomhead (1955) described a metatarsal rotation operation which he has used in the treatment of up to three prominent metatarsal heads. The offending metatarsal is divided half an inch from its base, the shaft is then resected subperiosteally and rotated through 180 degrees. Both these operations must be difficult especially in rheumatoid feet with fragile bone. Moreover, in many such patients the metatarsal heads are eroded and unsuitable for conservative surgery.

\section{SUMMARY}

1. An operation is described for the relief of irreversible claw toes associated with prominent and painful metatarsal heads.

2. The operation is suggested as an alternative to amputation of the toes.

3. The results of the operation done on twenty patients who have been followed up for a year or longer are reported.

I wish to thank Mr J. G. H. James, Mr H. J. Richards and Mr J. N. Wilson for allowing me to operate on their patients. I am grateful to Mr A. L. Eyre-Brook and Mr J. G. H. James for helpful criticism.

\section{REFERENCES}

Broomhead, R. (1955): Painful Feet. Rheumatism, 11, 19.

HoffmanN, P. (1912): An Operation for Severe Grades of Contracted or Clawed Toes. American Journal of Orthopaedic Surgery, 9, 441.

LaRmon, W. A. (1951): Surgical Treatment of Deformities of Rheumatoid Arthritis of the Forefoot and Toes. Quarterly Bulletin of Northwestern University Medical School, 25, 39.

MCKeever, D. C. (1952): Arthrodesis of the First Metatarsophalangeal Joint for Hallux Valgus, Hallux Rigidus and Metatarsus Primus Varus. Journal of Bone and Joint Surgery, 34-A, 129.

Nissen, K. I. (1957): In Operative Surgery, Vol. 5, p. 301. Edited by C. Rob and R. Smith. London: Butterworth \& Co. (Publishers) Ltd. 